\section{Prolonged response duration in lymphoma after rituximab maintenance therapy}

Treatment with a combination of rituximab and chemotherapy (R-chemotherapy) improves the prognosis of patients with follicular lymphomas (FLs) or mantle-cell lymphomas (MCLs). Forstpointner et al. have now demonstrated that maintenance therapy with rituximab (R-maintenance) after R-chemotherapy results in an improved response in lymphoma patients who are responsive to initial $\mathrm{R}$-chemotherapy.

The study included patients with refractory or recurring $\mathrm{FL}$ or $\mathrm{MCL}$ who were randomized to receive fludarabine, cyclophosphamide and mitoxantrone (FCM) alone or together with rituximab (R-FCM). High response rates were observed in the R-FCM arm and 176 of these responsive patients were subsequently randomized to receive $\mathrm{R}$-maintenance or no further treatment. All patients in the $\mathrm{R}$-maintenance arm had a prolonged response duration compared with patients who received no maintenance $(P=0.001)$, and this benefit of $R$-maintenance remained when the analysis was restricted to patients who had received initial R-FCM therapy $(P=0.035$ for those with FL and $P=0.049$ for those with MCL). The estimated percentages of patients alive at 3 years were $77 \%$ after R-maintenance therapy and $57 \%$ after no maintenance $(P=0.100)$.

These results demonstrate that the antilymphoma activity of rituximab is maintained through all phases of treatment, and indicate that R-maintenance is a promising therapy for patients with FLs and those with MCLs, producing no significant side effects.

Original article Forstpointner R et al. (2006) Maintenance therapy with rituximab leads to a significant prolongation of response duration after salvage therapy with a combination of rituximab, fludarabine, cyclophosphamide, and mitoxantrone (R-FCM) in patients with recurring and refractory follicular and mantle cell lymphomas: results of a prospective randomized study of the German Low Grade Lymphoma Study Group (GLSG). Blood 108: 4003-4008

\section{Whole-body PET-CT colonography proves accurate for colorectal cancer staging}

Colonography comprising a combined modality of PET and CT (PET-CT) could shorten the multimodality diagnostic work-up required for the conventional staging of patients with colorectal cancer. In a prospective study, Veit-Haibach et al. have demonstrated that the staging accuracy of whole-body PET-CT colonography is at least equivalent to that of CT followed by PET $(\mathrm{CT}+\mathrm{PET})$.

In total, 52 patients were referred for wholebody PET-CT colonography on the basis of clinical findings and optical colonoscopy. Of these patients, 47 (mean age 71 years) underwent surgery after PET-CT staging and were included in the study. On the basis of lesionto-lesion analysis, PET-CT colonography, CT alone (with a threshold for malignant lymph nodes of $0.7 \mathrm{~cm}$ ), and PET + CT correctly determined tumor-node-metastasis (TNM) stage in $74 \%, 52 \%$ and $64 \%$ of lesions, respectively. PET-CT colonography was significantly more accurate in defining TNM stage than CT imaging alone $(+22 \%, 95 \% \mathrm{Cl} 9-36 \% ; P=0.003)$, largely owing to a more accurate determination of the $\mathrm{T}$ stage. No significant difference in the accuracy of $\mathrm{N}$-stage determination was seen between PET-CT colonography and $\mathrm{PET}+\mathrm{CT}$. When compared with CT alone at a lymph node threshold of $1.0 \mathrm{~cm}$, PET-CT was significantly more accurate in determining malignant lymph nodes $(P=0.003)$. No statistically significant differences were found when comparing the M-staging accuracies of the different imaging modalities. Overall, when compared with conventional staging, PET-CT colonography altered the management of four patients. The changes in management were attributable to either a more accurate assessment of tumor stage or concomitant findings on PET-CT colonography.

\footnotetext{
Original article Veit-Haibach P et al. (2006) Diagnostic accuracy of colorectal cancer staging with whole-body PET/CT colonography. JAMA 296: 2590-2600
}

\section{Allogeneic hematopoietic stem cell transplantation increases risk of secondary malignancy}

Allogeneic hematopoietic stem cell transplantation (allo-HSCT) is a widely used therapeutic approach for patients with various life-threatening conditions; however, previous studies have shown that allo-HSCT can result in late undesired consequences such as secondary malignancy. Gallagher and Forrest carried out a study to determine the frequency 